\title{
AVALIAÇÃO DO NÍVEL DE CONHECIMENTO DE CONSUMIDORES DE PRODUTOS DIET E LIGHT QUE FREQUENTAM UM SUPERMERCADO DE CURITIBA
}

\section{EVALUATION OF THE KNOWLEDGE LEVEL ON DIET AND LIGHT PRODUCTS OF CONSUMERS AND CUSTOMERS ON A CURITIBA SUPERMAKET}

\author{
FERNANDA RORATO ${ }^{1}$, CLÁUDIA HELENA DEGÁSPARI ${ }^{2}$, FATIMA MOTTIN ${ }^{3}$
}

1 Aluna de Graduação do Curso de Nutrição da Universidade Tuiuti do Paraná. Rua Bento Viana, 947, apto 1101, Batel, 80240-110, Curitiba, PR.

e-mail: ferrorato @hotmail.com

2 Professora Adjunta da Universidade Tuiuti do Paraná.

e-mail: claudia.degaspari @utp.br

3 Professora Substituta da Universidade Federal do Paraná

e-mail: mottin_fatima@ yahoo.com.br_RECEBIDO: 15/08/106_ ACEITE: 05/09/06

\section{RESUMO}

Este artigo apresenta uma pesquisa que analisa o grau de conhecimento de consumidores de produtos diet e light vendidos em um supermercado de Curitiba. A amostra foi constituída de 80 clientes, selecionados aleatoriamente, entre homens e mulheres. Avaliou-se o grau de conhecimento entre os clientes através da aplicação de um formulário que apresentava perguntas abertas e fechadas sobre: renda familiar, grau de instrução, peso, altura, qual motivo faz uso dos produtos diet e light, grau de conhecimento sobre a diferença de cada produto e onde obteve informações sobre os produtos. Os resultados indicam que os entrevistados se concentram entre 15 e 44 anos, somando 75\%; a maioria, 58\%, consomem produtos Light e 33\% consomem os dois produtos. Diet é o produto menos consumido. Os maiores consumidores são as mulheres, representando $73 \%$ dos entrevistados. Em relação à renda mostram o esperado, que produtos light e diet são mais consumidos por pessoas que tem renda familiar alta. A maioria dos entrevistados se classificam pelo índice de massa corpórea na classe de eutrofia. Dentre os entrevistados observa-se que 36\% informações que se obtêm sobre estes produtos advêm de revistas. Conclui-se que não depende de renda, de instrução, de sexo ou idade o conhecimento sobre esses produtos. $O$ que influencia esse conhecimento, segundo os dados, é procurar a informação no 
lugar certo. As pessoas consomem os produtos sem terem certeza do por que usam ou para que servem.

Palavras-chave: Produtos diet ; produtos light ; consumidor

\begin{abstract}
This article presents a research that analyzes the consumers' knowledge degree about diet and light products sold in a supermarket of Curitiba. The sample was constituted of 80 customers, randomly selected, among men and women. The knowledge degree was evaluated among the customers through the application of a form with open and closed questions about pay family, instruction degree, weight, height, reason to make use of diet and light products, knowledge degree on the difference between these products and source of information about them. The results indicate that the interviewees range between 15 and 44 years (75\%), most of them (58\%) consume light products, and 33\% consume both type of products. Diet is the less consumed product. Most part of consumers is women, representing $73 \%$ of the interviewees. In relation to the income they showed the expected, that light and diet are more consumed by people with higher income. Most of the interviewees are classified by the index of corporal mass as eutrofia. It's observed that $36 \%$ of the interviewees obtain information on these products in magazines, and it was concluded that the knowledge on these products don't depend on income, instruction degree, sex or age. According to the data, the source of information influences that knowledge. People consume these products without being sure of the reason to use them.
\end{abstract}

Key-words: Diet products ; light products; consumer

\title{
1 INTRODUÇÃO
}

A busca por uma alimentação alternativa é, sem dúvida, uma realidade nos dias de hoje. Consumidores que buscam novos e saudáveis hábitos alimentares têm nos produtos diet e light grandes aliados. Os mais jovens procuram os produtos geralmente com preocupações ligada à estética corporal e, entre os mais velhos, a procura prende-se à preservação ou manutenção da boa saúde (LERAYER et al, 1998).

Cada vez mais, encontramos produtos com características diversas nas prateleiras do supermercado:" Isento de açúcar"; "Sem sódio"; "Teor reduzido de 
gorduras", entre outros. Essas informações precisam estar dispostas de forma clara e legível para os consumidores (ABIAD, 2005).

Os rótulos são a maior fonte de informações quanto à composição, qualidade e características dos alimentos e auxiliam para uma escolha certa e adequada na hora da compra (ABIAD, 2005).

Alimentos diet são aqueles especialmente formulados e/ou produzidos de forma que sua composição atenda necessidades dietoterápicas especificas de pessoas com exigências físicas, metabólicas, fisiológicas e/ou patológicas particulares. Com restrição de açúcares (mono e dissacarídeos), sódio, gorduras, colesterol, aminoácidos ou proteínas, dietas para controle de peso, administradas por sonda, e modificadas em sua estrutura (CANDIDO \& CAMPOS, 1995).

O alimento diet por exemplo, nem sempre é um alimento "sem açúcar"; na maioria das vezes há restrição de algum nutriente, seja esse carboidrato (açúcares), gordura, proteína ou outros. O mesmo é destinado às pessoas que não podem ou não querem ingerir determinado nutriente, podendo ser consumido por qualquer pessoa (ABIAD,2005, TEIXEIRA NETO, 2003).

O termo light é utilizado para alimentos produzidos de forma que sua composição reduza em, no mínimo, 25\% do valor calórico e/ou alguns nutrientes como: açúcares, gorduras saturadas, gorduras totais, colesterol e sódio, quando comparado ao valor normal (INMETRO, 2004).

Portanto, com a retirada ou redução de algum nutriente, o alimento pode apresentar uma diminuição de calorias, e o consumidor que deseja emagrecer deverá estar atento à tabela nutricional, que é obrigatória, verificando se a redução é significativa e se justifica a substituição do alimento convencional por estes produtos, uma vez que costumam ser mais caros que os convencionais (INMETRO, 2004).

Os consumidores vão às compras buscando qualidade, preço, comodidade, segurança, conforto, praticidade e saúde. Hoje $20 \%$ das donas de casa brasileiras estão preocupadas com a saúde da família e aproximadamente $35 \%$ dos domicílios consomem algum tipo de produto light e/ou diet (ABIAD, 2005). 
Tendo em vista que o conceito de produtos diet e light não está bem definido na população em geral, o presente estudo objetiva avaliar o nível de conhecimento de pessoas consumidoras de produtos diet e light que freqüentam o supermercado Mercadorama da av. Sete de Setembro na cidade de Curitiba.

\section{METODOLOGIA}

Realizou-se a coleta de dados em um supermercado de classe média alta, no Mercadorama da Avenida Sete de Setembro localizado na região de Curitiba.

A coleta de dados foi realizada através da aplicação de um formulário (Anexo 1), que compreende 10 perguntas fechadas e fechadas simples, onde foi avaliado o conhecimento de pessoas consumidoras de produtos diet e light; a coleta de dados foi realizada em 2 dias, uma terça-feira e uma sexta-feira do mês de outubro de 2005 , dias de maior movimento do supermercado, e os dados foram tabulados e analisados através de análise estatística descritiva.

A pesquisa foi realizado em um supermercado onde se tem em média 3200 clientes por dia. Com esse tamanho de população, dada uma confiança de 95\% na amostragem por proporção com uma margem de erro de 5\%, a amostra necessária é de 343 respondentes. Sendo o interesse da pesquisa apenas consumidores de produtos light e diet, e como é restrito esse público, acredita-se que cerca de $24 \%$ desse tamanho de amostra seja suficiente para termos uma estimativa confiável do objetivo da pesquisa. Então se estipulou uma amostragem intencionada de conseguir 80 clientes que consumam produtos light e diet (TRIOLA, 1999; AMERINE,1965).

Para análise foram utilizados além de gráficos e tabelas, o Teste QuiQuadrado $\left(\chi^{2}\right)$ (TRIOLA, 1999). 


\subsection{TECNICA ESTATÍSTICA UTILIZADA PARA ANÁLISE}

O Teste Qui-Quadrado $\left(\chi^{2}\right)$ é aplicável em dados representados em forma de freqüência para detectar significância estatística da diferença entre dois grupos independentes.

Estatística do teste $\Rightarrow \chi^{2}=\frac{\sum(\mathrm{O}-\mathrm{E})^{2}}{\mathrm{E}} \quad \begin{aligned} \text { onde: } \mathrm{O} & =\text { Freqüência observada } \\ \mathrm{E} & =\text { Freqüência esperada }\end{aligned}$

O Teste $\chi 2$ (teste Qui-Quadrado) é uma estatística não paramétrica de fácil compreensão e muito usada para verificar se há diferença entre freqüências esperadas e freqüências observadas de duas ou mais categorias. No caso, a freqüência esperada é sempre aquela que determina a igualdade dos grupos. $O$ teste conflita a freqüência esperada da observada, e se der diferença significativa entre as duas categorias, os grupos são considerados diferentes.

Este teste compara o valor encontrado pela estatística do teste com um valor tabelado, conforme o nível de confiança estabelecido pelo pesquisador, ou se for usado algum software, usa-se para uma confiança de $95 \%$ que:

- $p$-valor < 0,05: Existe diferença significativa entre as classes, o que afirma que uma classe é melhor ou maior que a outra.

- p-valor > 0,05: Não existe diferença significativa entre as classes, o que impossibilita de dizer se uma classe é melhor do que a outra (TRIOLA, 1999).

\section{ANÁLISE E INTERPRETAÇÃO DOS RESULTADOS}

A amostragem realizada foi de 80 clientes de produtos light e diet, os quais se caracterizam da seguinte forma:

- O Gráfico 1 mostra que a maioria dos entrevistados na amostra, 58\% consomem produtos Light e $33 \%$ consomem os dois produtos. Diet é o produto menos consumido. 


\section{GRÁFICO 1: PRODUTO CONSUMIDO}

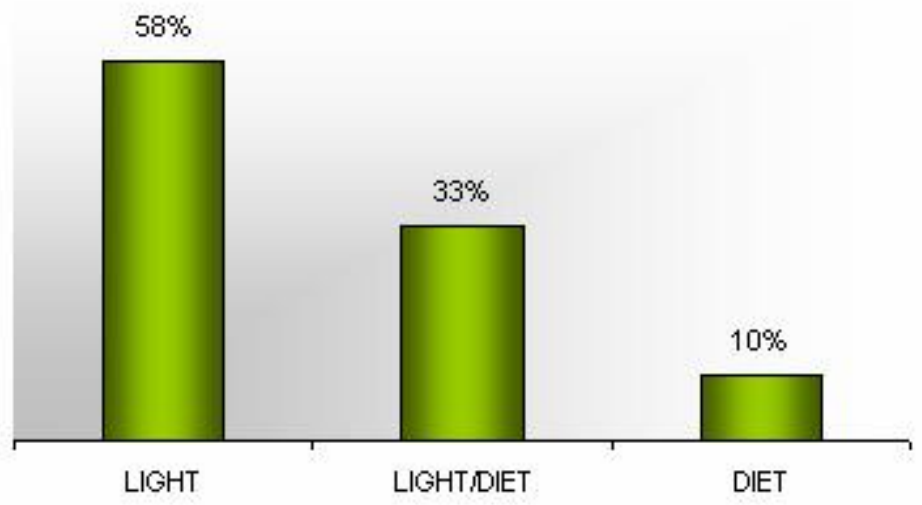

- Os entrevistados se concentram entre 15 e 44 anos, somando $75 \%$, mostrando que o consumo desses produtos por pessoas mais idosas é mais baixo que em uma faixa etária mais jovial, isso é visualizado no Gráfico 2.

TABELA 1: CRUZAMENTO ENTRE FAIXA ETÁRIA E CONSUMO EM \%

\begin{tabular}{|c|c|c|c|c|}
\hline IDADE & LIGHTDIET & DIET & LIGHT & TOTAL \\
\hline 15 A 24 AHOS & $15 \%$ & $19 \%$ & $22 \%$ & $21 \%$ \\
\hline 25 A 34 AHOS & $31 \%$ & $50 \%$ & $41 \%$ & $34 \%$ \\
\hline 35 A 44 AHOS & $27 \%$ & $19 \%$ & $13 \%$ & $20 \%$ \\
\hline 45 A 54 AHOS & $15 \%$ & $13 \%$ & $7 \%$ & $11 \%$ \\
\hline ACIMA DE 55 AHOS & $8 \%$ & $0 \%$ & $17 \%$ & $13 \%$ \\
\hline SEM RESPOSTA & $4 \%$ & $0 \%$ & $0 \%$ & $1 \%$ \\
\hline TOTAL & $100 \%$ & $100 \%$ & $100 \%$ & $100 \%$ \\
\hline
\end{tabular}

\section{GRÁFICO 2. IDADE DOS ENTREVISTADOS}

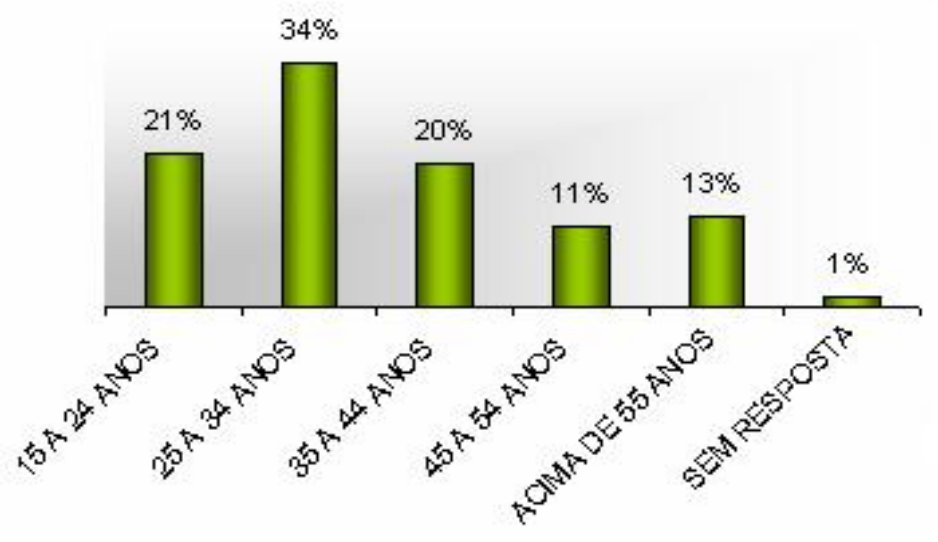


- Cruzando idade com produtos, observa-se que existe uma tendência de uma faixa etária versus produto apenas no consumo do produto light na faixa de 25 a 34 anos, nesse caso podemos afirmar que o público alvo do produto light são pessoas nessa faixa etária (essa diferença é comprovada pelo Teste Qui Quadrado, com uma confiança de $99 \%$ e um p-valor de 0,003). Nos produtos diet o teste não pôde ser aplicado porque as exigências não foram cumpridas (poucos dados nas classes) e as pessoas que consomem os dois produtos não têm um padrão definido em relação à idade.

- Em relação ao sexo, os maiores consumidores são as mulheres, representando $73 \%$ dos entrevistados.

\section{GRÁFICO 3: SEXO DOS ENTREVISTADOS}

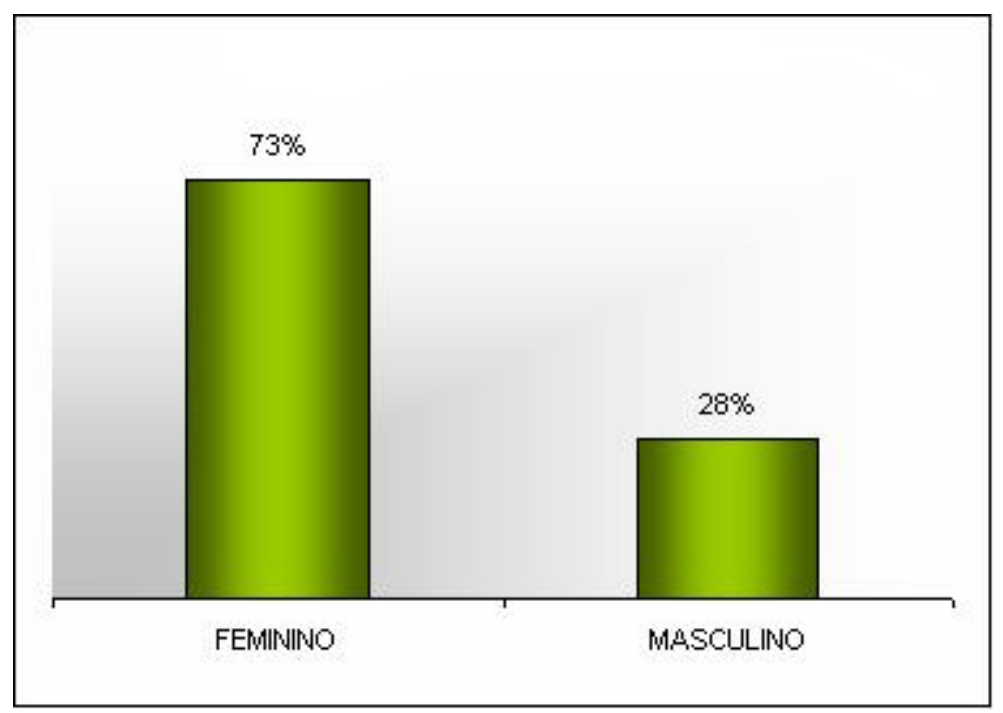

- Dentro de cada sexo, observa-se o mesmo comportamento. A tabela 2 demonstra que, tanto homens quanto mulheres, consomem mais os produtos light (essa conclusão pode ser assumida com um nível de confiança de 99\% e um p-valor menor que 0,001, com base no Teste Qui-Quadrado, para os dois sexos). 
TABELA 2: CONSUMO DOS PRODUTOS DENTRO DE CADA SEXO

\begin{tabular}{c|c|c|c|c}
\hline SEXO & LIGHTDIET & DIET & LIGHT & TOTAL \\
\hline FEMIHIHO & $36 \%$ & $10 \%$ & $53 \%$ & $100 \%$ \\
MASCULIHO & $23 \%$ & $9 \%$ & $68 \%$ & $100 \%$ \\
\hline TOTAL & $33 \%$ & $10 \%$ & $58 \%$ & $100 \%$ \\
\hline
\end{tabular}

Em relação à renda o comportamento dos dados, visualizado no Gráfico 4, mostram o esperado, que produtos light e diet são mais consumidos por pessoas que tem renda familiar alta, no caso acima de 6 salários mínimos encontram-se $77 \%$ dos entrevistados. E pode-se assumir que a faixa que mais compra são os que ganham acima de 8 salários mínimos, também comprovados com o Teste Qui-Quadrado, com uma confiança acima de 95\% ( $p$-valor menor que 0,0001).

\section{GRÁFICO 4: RENDA DOS ENTREVISTADOS.}

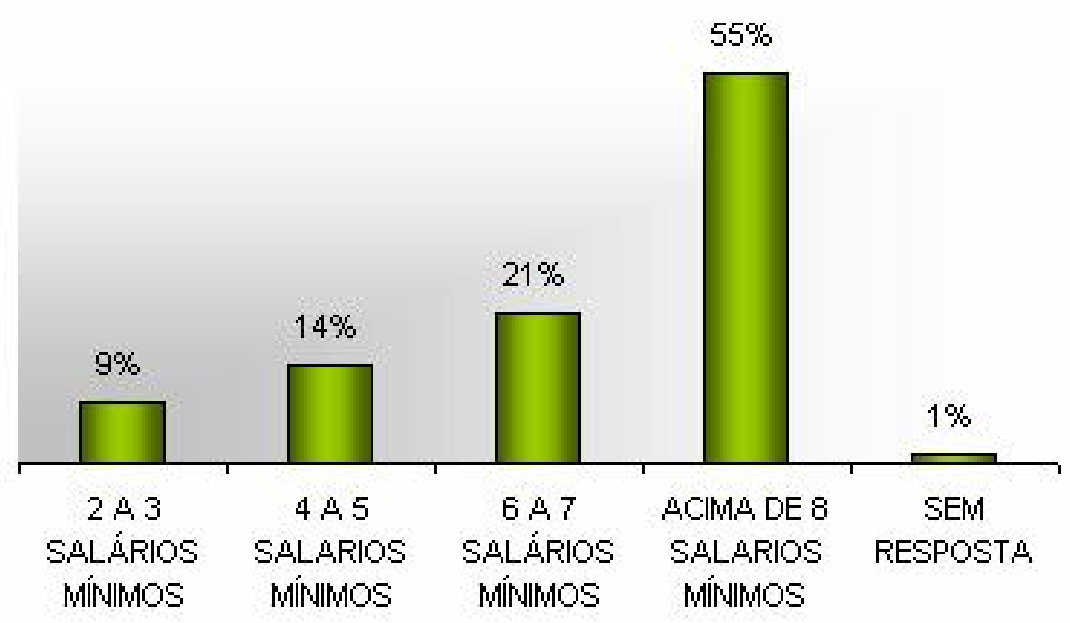

- O grau de instrução dos entrevistados é elevado (observe Gráfico 5), sendo que 70\% está cursando ou já concluiu o ensino superior. Pode-se afirmar que o público alvo desses produtos são pessoas com $3^{\circ} \mathrm{grau}$, com uma confiança acima de 99\% (p-valor menor que 0,001), através do teste Qui-quadrado. 


\section{GRÁFICO 5: GRAU DE INSTRUÇÃO DOS ENTREVISTADOS.}

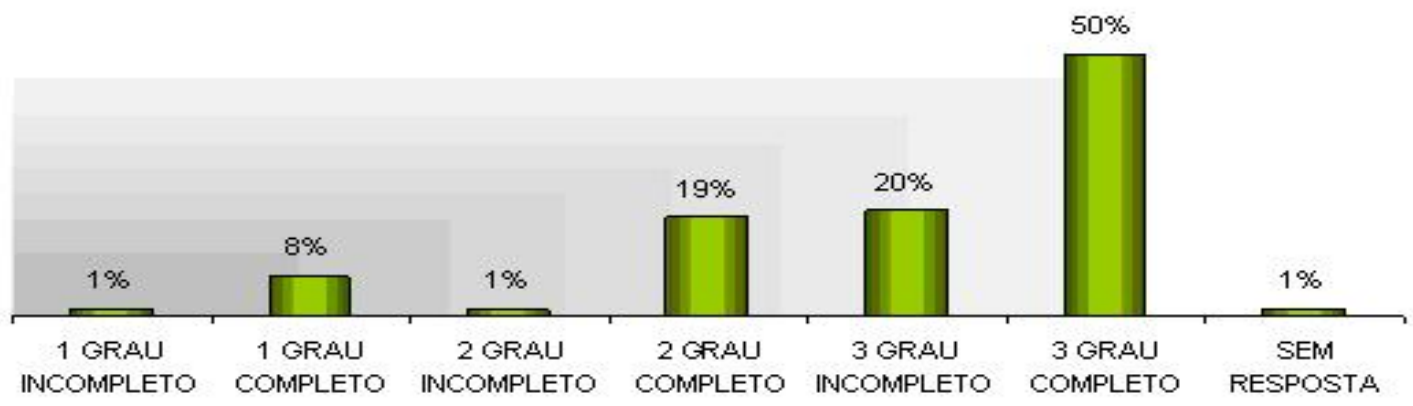

- Os entrevistados estão descritos em relação a peso e altura, em média, segundo a tabela abaixo (tabela 3).

TABELA 3: DISTRIBUIÇÃO DO PESO E DA ALTURA

\begin{tabular}{c|c|c|c|c}
\hline & \multicolumn{2}{|c|}{ PESO IKG } & \multicolumn{2}{c}{ AL TURA I CM } \\
\hline SEXO & MÉDIA & DESUIO & MÉDIA & DESUIO \\
\hline FEMIHIHO & 60 & 10 & 164 & 7 \\
MASCULIHO & 82 & 9 & 181 & 7 \\
\hline TOTAL & 66 & 13 & 168 & 10 \\
\hline
\end{tabular}

Completando a informação de peso e altura, a maioria dos entrevistados se classificam pelo índice de massa corpórea na classe de eutrofia.

\section{GRÁFICO 6: CLASSIFICAÇÃO IMC}

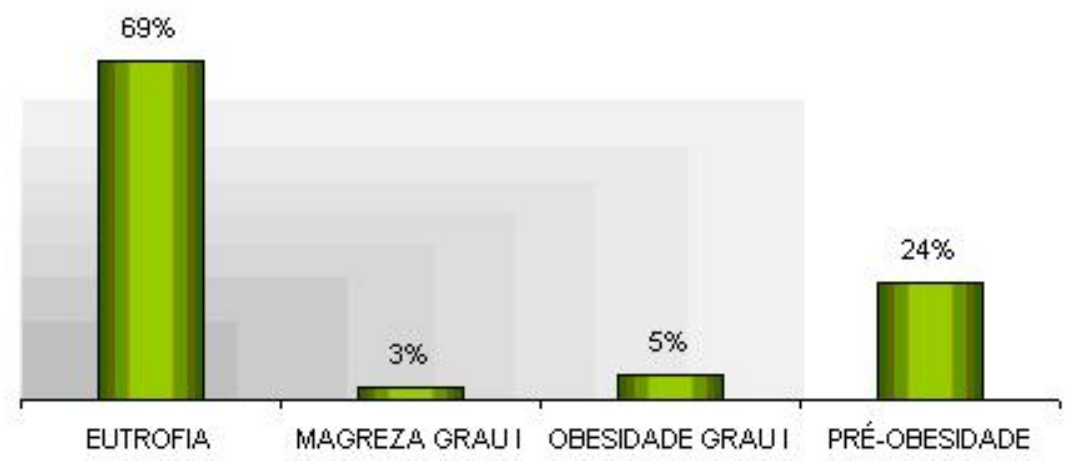


- Sobre as razões para uso destes produtos foram obtidas as respostas visualizadas nos Gráficos 7 e 8:

Para os dois tipos de produtos observa-se que a atribuição de "produtos saudável" é a mais citada, embora apenas no produto light pode-se afirmar que essa é uma característica que a maioria cita, pois o Teste do Qui-Quadrado tem um $p$ valor de 0,001 .

\section{GRÁFICO 7: NA OPINIÃO DOS ENTREVISTADOS SOBRE PARA QUE SERVE O PRODUTO DIET.}

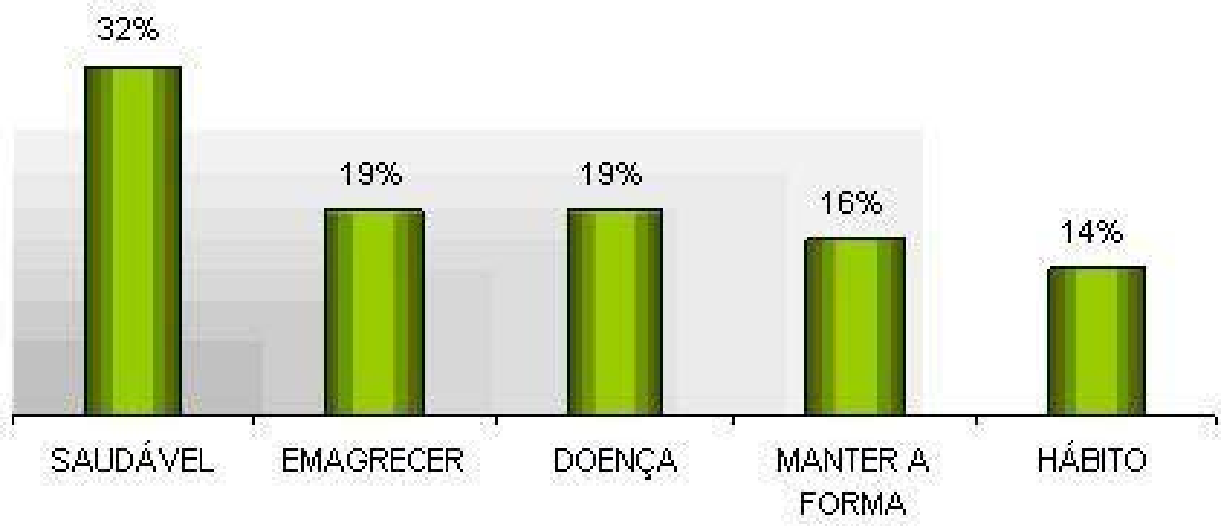

No produto diet a atribuição "saudável" foi a característica mais citada, porém não em tão grande freqüência como no light, não apresentando um Teste Qui-Quadrado significativo. Isto leva a concluir que quem usa o produto diet não tem uma opinião definida sobre o por que ele usa. 


\section{GRÁFICO 8: NA OPINIÃO DOS ENTREVISTADOS PARA QUE SERVE O PRODUTO LIGHT.}

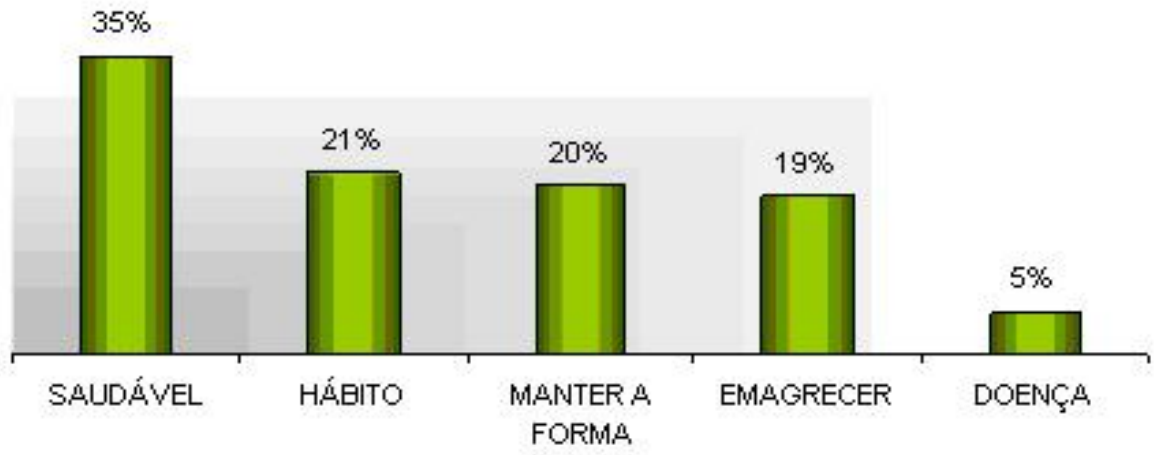

- O objetivo do questionário é comprovar se as pessoas conhecem ou não para que servem os produtos light e diet. O Gráfico 9 abaixo é resultado da avaliação e da conversa com os entrevistados. Observando o gráfico tem-se que está bem dividida a resposta "sim" e "não", e é comprovado pelo Teste Qui-Quadrado que não existe diferença significativa entre essas duas respostas ( $p$-valor igual 0,26). Não podemos afirmar que os entrevistados consomem o produto pelos seus reais motivos, ou ainda que eles não sabem definir o por que eles estão consumindo.

\section{GRÁFICO 9: OS ENTREVISTADOS SABEM SE EXISTE DIFERENÇA ENTRE} LIGHT E DIET.

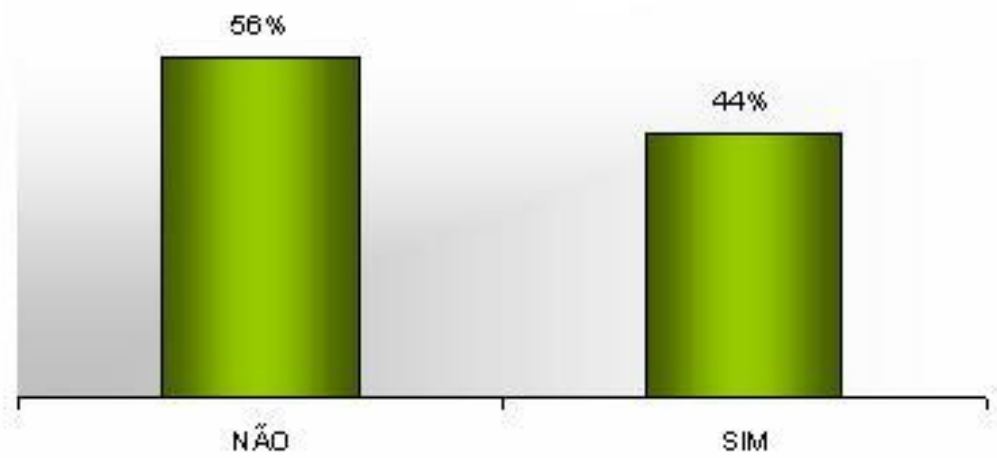


No geral tem-se que não existe diferença entre a freqüência dos consumidores que sabem o porque usam e os que não sabem. O que se afirma é que as pessoas estão confusas quando tentam definir o porque do uso dos produtos.

Avaliando cada produto observa-se esse mesmo comportamento entre consumidores de um ou de outro produto; entre os que consomem os dois (light/diet), é comprovado com 95\% de confiança (p-valor de 0,04) que eles não sabem para que serve o produto que eles consomem, conforme demonstrado na Tabela 4.

TABELA 4: COMPARAÇÃO DO USO DO PRODUTO COM O SE SABE E PRA QUE SERVE

\begin{tabular}{c|c|c|c}
\hline PRODUTO & HAO & SIM & TOTAL \\
\hline LIGHT DIET & $69 \%$ & $31 \%$ & $100 \%$ \\
DIET & $38 \%$ & $63 \%$ & $100 \%$ \\
LIGHT & $52 \%$ & $48 \%$ & $100 \%$ \\
\hline TOTAL & $56 \%$ & $44 \%$ & $100 \%$ \\
\hline
\end{tabular}

Outra pergunta avaliada e mostrada no Gráfico 10, é por qual meio de comunicação eles recebem informações sobre produtos light e diet.

\section{GRÁFICO 10: INFORMAÇÕES SOBRE O USO DOS PRODUTOS}

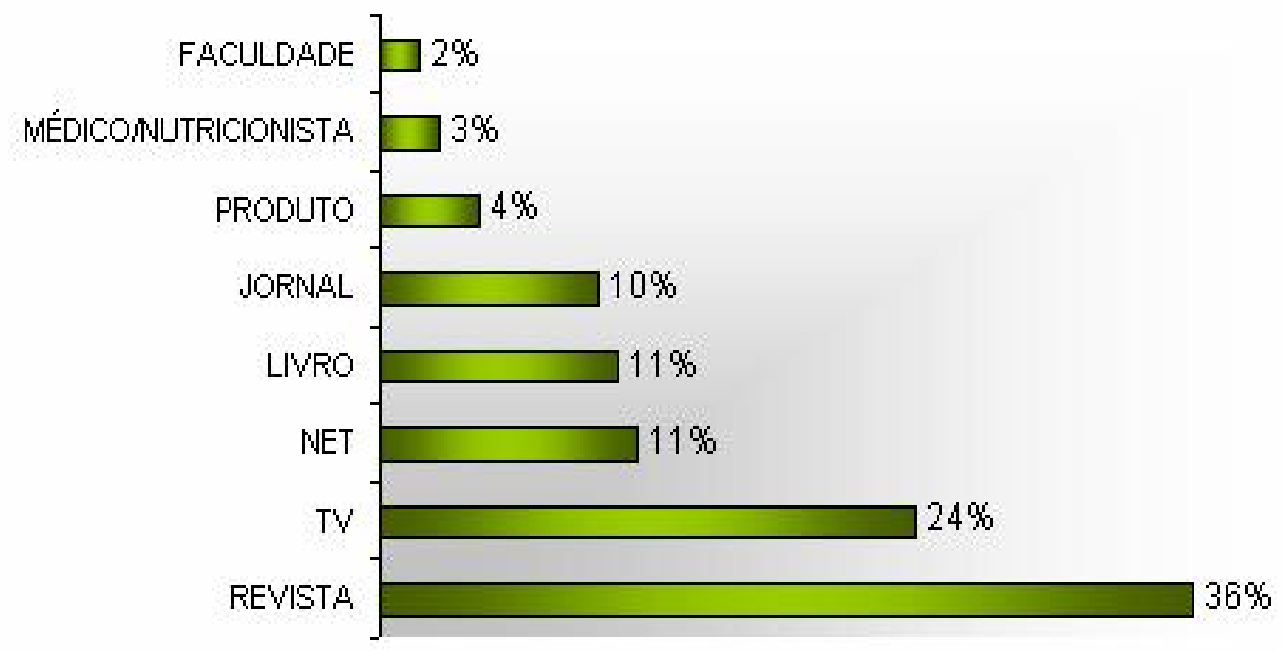


As maiorias dos entrevistados citaram as revista como o principal meio de informação sobre os produtos.

Fazendo o cruzamento dos meios de informação (Gráfico 11) com a resposta da pergunta sobre a finalidade do produto, tem-se dos itens mais citados na revista, internet, livro e tv o Teste Qui-Quadrado com as seguintes conclusões:

- Revista: Teste não significativo, com um p-valor de 0,1599. Entre os entrevistados que dizem se informar por revista, alguns sabem e outros não a diferença; não existe distinção entre o "sim" e o "não";

- Internet: Teste significativo com um p-valor de 0,0522 com um nível de significância acima de 90\%. Entre os entrevistados que dizem se informam por internet, pode-se afirmar que demonstram saber a diferença entre os produtos;

- Livro: Teste significativo com um p-valor de 0,003 com um nível de significância acima de 99\%. Entre os entrevistados que dizem se informar por livro, pode-se afirmar que demonstram saber a diferença entre os produtos;

- TV: Teste significativo com um p-valor de 0,0124 com um nível de significância acima de 95\%. Os entrevistados que dizem se informar por TV, pode-se afirmar que demonstram não saber a diferença entre os produtos.

\section{GRÁFICO 11: INFORMAÇÕES SOBRE O USO DOS PRODUTOS VERSUS O MEIO DE INFORMAÇÃO.}

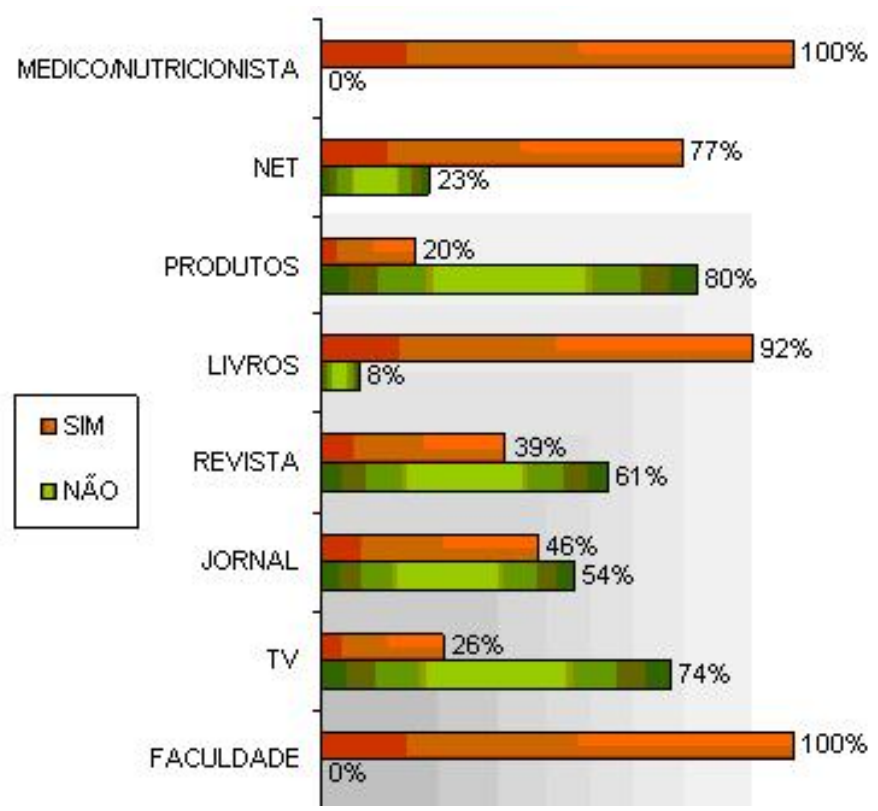


Os cruzamentos foram feitos de todas as formas: com renda, instrução, sexo, porém apenas se encontrou alguma influência na resposta "sim" e "não", nos meios de informação citados acima e nos consumidores dos dois produtos.

O que nos leva a concluir no trabalho que não depende de renda, de instrução, de sexo ou idade o conhecimento sobre esses produtos. O que influencia esse conhecimento, segundo os dados, é procurar a informação no lugar certo.

\section{CONCLUSÃO}

A pesquisa dos produtos foi realizada entre 80 clientes que freqüentam um supermercado localizado na região de Curitiba.

O perfil dos clientes participantes apresentou as seguintes características: $58 \%$ dos entrevistados consomem produtos light a maioria dos clientes era do sexo feminino com $73 \%$ do total, mas observa-se que no geral entre homens e mulheres os dois consomem mais os produtos light; $34 \%$ dos clientes apresentavam idades entre 25 e 34 anos; e 55\% tinham uma renda mensal acima de 8 salários mínimo. Em relação ao grau de instrução pode-se afirmar que o publico alvo desses produtos são pessoas com $3^{\circ} \mathrm{grau}$, com uma confiança de 99\% ( $p$-valor menos que 0,001).

Para poder classificar os participantes em relação ao índice de massa corpórea foi perguntado o peso e altura de cada um deles, onde $69 \%$ estão eutróficos. Observa-se também que não podemos afirmar que os entrevistados consomem o produto pelos seus reais motivos, ou ainda que eles não sabem definir o por que eles estão consumindo, o que se afirma é que as pessoas estão confusas quando tentam definir o por que do uso dos produtos. Analisando cada produto tem-se esse mesmo comportamento nos consumidores de um ou de outro produto, os que consomem os dois (light/diet), é comprovado com $95 \%$ de confiança ( $p$-valor de 0,04 ) que eles não sabem para que serve o produto que eles consomem. Outro indicador disso é sobre aonde os participantes obtiveram as informações sobre os produtos, que as pessoas que se interessam por internet e 
livros sabem diferenciar os produtos e as que lêem revistas e TV não sabem diferenciar um produto light de um diet.

O mais preocupante é que as pessoas consomem os produtos diet e light sem terem certeza do por que usam ou para que servem.

\section{REFERÊNCIAS}

ABIAD. Diet e Light. Disponível em: http://www.abiad.gov.br. Acesso em: 13 out 2005.

AMERINE, Maynard A.; PANGBORN, Rose M.; ROESSLER, Edward B. Principles of sensoy evaluation of food. London: Academic Press, 1965.

BENNETT, J. Claude et al. Cecil Tratado de medicina interna. Rio de Janeiro: Guanabara, 1997.

CÃNDIDO, Lys Mary Bileski, CAMPOS, Adriane Mulinari. Alimentos para fins especiais: Dietéticos. São Paulo: Varela, 1995.

CUPPARI, Lílian. Guia da nutrição: Nutrição clínica no adulto. São Paulo: Manole, 2002.

INMETRO. Produtos Diet e Light - parte I e II. Disponível em: http://www.inmetro.gov.br/consumidor/produtos. Acesso em: 17 mai 2005.

LERAYER, Alda Luiza Santos et al. Nova legislação de produtos lácteos e de alimentos para fins especiais, diet, light e enriquecidos. São Paulo: Fonte, 1998.

MAHAN, L. Kathleen, STUMP - ESCOT Sylvia. Krause: Alimentos, nutrição e dietoterapia. 9.ed. São Paulo: Roca, 1998.

TEIXEIRA NETO, Faustino. Nutrição clínica. Rio de Janeiro: Guanabara, 2003.

TRIOLA, Mario. Introdução à Estatística. 7.ed. São Paulo: L.T.C.,1999.

WAITZBERG, Dan Linetzky, Nutrição oral, enteral e parenteral na prática clínica. 3.ed. São Paulo: Atheneu, 2000.

WILLIAMS, Sue Rodwell. Fundamentos de nutrição e dietoterapia. 6.ed. Porto Alegre: Artmed, 1997. 
ANEXO 1 - FORMULÁRIO APLICADO NA PESQUISA

1.VOCÊ É CONSUMIDOR DE PRODUTOS DIET, LIGHT OU OS DOIS?

$\begin{array}{lll}\text { () DIET ( ) LIGHT } & \text { ( ) DIET E LIGHT }\end{array}$

2. IDADE

() 15 A 24 ANOS () 25 A 34 ANOS ()35 A 44 ANOS () 45 A 55 ANOS () >55 ANOS

3. SEXO: ( ) FEMININO ( ) MASCULINO

4. QUAL É A SUA RENDA FAMILIAR?

() 1 S.M. ( )2 a 3 S.M. () 4 a 5 S.M. () 6 a 7 S.M. () ACIMA DE 8 S.M.

5. QUAL O SEU GRAU DE INSTRUÇÃO?

() $1^{\circ} \mathrm{GRAU}$ INCOMPLETO () $1^{\circ}$ GRAU COMPLETO () $2^{\circ}$ GRAU INCOMPLETO

() $2^{\circ} \mathrm{GRAU}$ COMPLETO () $3^{\circ} \mathrm{GRAU}$ INCOMPLETO () $3^{\circ} \mathrm{GRAU}$ COMPLETO

( ) NENHUMA FORMAÇÃO

6. QUAL É O SEU PESO : PESO ___ ALTURA IMC : $\mathrm{kg} / \mathrm{m}^{2}$

7. PORQUE VOCÊ FAZ USO DE PRODUTOS :

DIET

() PARA EMAGRECER

() PARA MANTER A FORMA

() POR ALGUMA DOENÇA

() POR HÁBITO

() POR SER MAIS SAUDÁVEL

() OUTROS
LIGHT

() PARA EMAGRECER

() PARA MANTER A FORMA

() POR ALGUMA DOENÇA

( ) POR HÁBITO

() POR SER MAIS SAUDÁVEL

() OUTROS

8. VOCÊ SABE A DIFERENÇA DE UM PRODUTO DIET DE UM PRODUTO LIGHT?
() SIM
( ) NÃO

9. ONDE OBTEVE AS INFORMAÇÕES SOBRE ESSES PRODUTOS?
() TV
( ) LIVROS

() OUTROS 